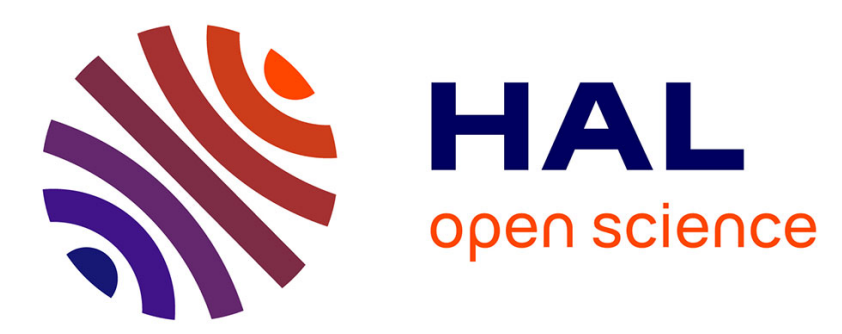

\title{
Evaluating Ground Risk for Road Networks Induced by UAV Operations
}

Sylvain Bertrand, Nicolas Raballand, Flavien Viguier

\section{To cite this version:}

Sylvain Bertrand, Nicolas Raballand, Flavien Viguier. Evaluating Ground Risk for Road Networks Induced by UAV Operations. 2018 International Conference on Unmanned Aircraft Systems (ICUAS), Jun 2018, Dallas, United States. 10.1109/ICUAS.2018.8453441 . hal-02421330

\section{HAL Id: hal-02421330 \\ https://hal.science/hal-02421330}

Submitted on 20 Dec 2019

HAL is a multi-disciplinary open access archive for the deposit and dissemination of scientific research documents, whether they are published or not. The documents may come from teaching and research institutions in France or abroad, or from public or private research centers.
L'archive ouverte pluridisciplinaire HAL, est destinée au dépôt et à la diffusion de documents scientifiques de niveau recherche, publiés ou non, émanant des établissements d'enseignement et de recherche français ou étrangers, des laboratoires publics ou privés. 


\title{
Evaluating Ground Risk for Road Networks Induced by UAV Operations*
}

\author{
S. Bertrand, N. Raballand and F. Viguier
}

\begin{abstract}
This paper presents a new method to evaluate the risk for road networks induced by UAV operations. The hazardous event considered for risk assessment is an accident with damages caused by an UAV falling on a road. Computation models are proposed to evaluate the probability of each event the sequence of which would lead to such an accident. Influences of the type of road as well as the road traffic are taken into account in the models. Simulation results are presented to illustrate risk evaluation by the proposed approach through a case study of a road network of several kilometres. Some metrics are also introduced to analyse the risk in a comprehensive way.
\end{abstract}

\section{INTRODUCTION}

There is a growing interest in enabling Unmanned Aerial Vehicles (UAVs) to perform outdoor Long Range Operations (LRO) such as inspection of power lines, monitoring of transportation networks, etc. Nevertheless, business opportunities would exist for such a civilian market only if the flights can be performed as a fully operational process and not as isolated flight experiments. This requires flight authorizations to be easily and quickly obtained and thus a good mastering of the risk levels associated to the operation (potential severity, exposition time to the risk, etc.).

Risks can be decomposed into aerial and ground classes. The first class encompasses risks of air collisions with other users of the airspace, cooperative or non-cooperative. Lowaltitude airspace should be considered as UAVs are to fly close to the ground for such LRO missions (basically below $150 \mathrm{~m}$ Above Ground Level in France according to current regulations). The second class gathers ground risks with respect to people of inhabited areas and to infra structures including critical infrastructures (e.g. power plants, classified areas, etc.) but also infrastructures for which the inspection mission is intended or that are located close to them. In the case of railways inspection missions for example, portions of the tracks are often located close to other linear infrastructures such as power lines or roads.

A risk analysis should therefore consider - at least - all these dimensions to guarantee safe operations. A lot of work has been done in the literature to provide methodologies that can

*This work has been supported by the research partnership between SNCF Réseau, Altametris and ONERA.

S. Bertrand and N. Raballand are with ONERA - The French Aerospacelab, Palaiseau, France (sylvain.bertrand@onera.fr, nicolas.raballand@onera.fr).

F. Viguier is with Altametris, Paris, France (flavien.viguier@altametris.com). be used to quantitatively assess some of these risks through probabilistic risk assessment. This is the case for mid-air collision risks between two given flying vehicles [1][2][3]. This is also the case for ground risk assessment for people of inhabited areas [4][5]. A very good overview of existing approaches and comparison of the risk models developed can be found in [6]. This type of approaches has been used to assess the risk of UAV operations such as power line inspection [7] or railways monitoring [8].

To the authors' knowledge, there exists no work in the literature that provides models to evaluate the ground risk for road networks during UAV operations. Therefore the main contribution of this paper is to propose such models to evaluate the risk of an accident (with damage) to a user of a road network (vehicle and/or passengers), due to an impact with an UAV descending to a road. Fixed-wing UAVs are considered in this paper and not rotorcraft-based UAVs, due to the Long Range characteristic of the considered missions. These proposed models can be used in a probabilistic risk assessment approach in the same way as risk models regarding people of inhabited areas. Although some similarities may exist between the models for these two risk categories (e.g. need for computation of a probability of impact at ground on a specific location), there exist some strong specificities regarding risk models for road networks. This is mainly due to the nature of the associated data (dependence on the type of road, on road speed and traffic, etc.).

The paper is organized as follows. The risk considered is described in the next section along with the main risk equation. Section III presents the data used to describe the road network and the proposed models that will be used to represent a vehicle and road traffic. Risk models are then proposed in Section IV for the evaluation of all the terms of the risk equation. A simulation example is proposed in Section $\mathrm{V}$ to illustrate the use of the proposed models for risk assessment performed in Section VI. Concluding remarks are finally provided in the last section of the paper.

\section{DEFINITION OF THE RISK TO BE EVALUATED}

A definition of the risk in consideration is provided first in this section. Decomposition into a sequence of hazardous events is then described.

\section{A. Risk definition}

In this paper, we consider the risk of getting an accident with damage for a user of a road network. The term user can be instantiated as "vehicle" and/or "passengers" of the vehicles depending on the evaluation that is required. It is 
assumed that the accident is caused by a direct impact between a fixed-wing UAV heading to the road and the vehicle. Only a direct accident is considered, assuming that all the drivers respect stopping distances between vehicles and therefore that an impact between the UAV and a first vehicle will result in no "induced" accidents between this vehicle and others on the road. This may seem to be a very strong assumption, but it can be relaxed by performing a complementary risk study to the one of interest in this paper to evaluate the risk of induced accidents between vehicles on the road. This can be addressed by classical road accident methods (eg. [9]) and is beyond the scope of this paper.

\section{B. Risk decomposition}

Similarly to risk analysis regarding casualties for people of inhabited areas, the hazardous event of "getting an accident" with damage for a user of the road network is decomposed into a succession of four events which are (see Figure 1):

- Loss of control of the UAV leading to a non-controlled descent to the ground.

- Impact on a road in a non-controlled way. It excludes emergency landings for example.

- Collision with a vehicle. Although different types of vehicles should be considered, in this paper analysis is performed by focusing on cars (see Section III-A).

- Damages to user. Depending on the purpose of the risk analysis, "user" can be defined as "vehicle" and/or "passenger" of a vehicle.

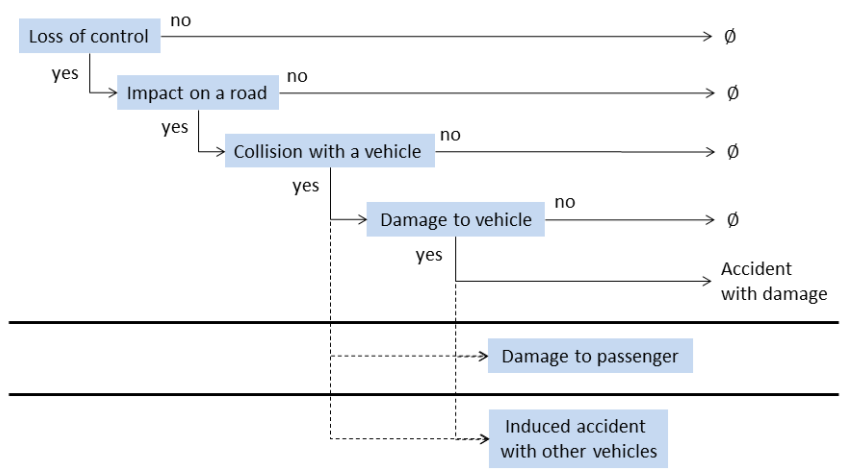

Figure 1. Sequence of events leading to an accident with damage (as considered within the scope of this paper)

Note that landing or impacting on a road in a controlled way can also lead to some accident. This case is not explicitly considered in this paper but can be handled by the proposed method by modifying the term "Loss of control" and the underlying model or probability assumption accordingly.

Conditionality in the realization of the events of this sequence leads to the following probabilistic expression of the risk:

$$
\begin{aligned}
\operatorname{Pr}\{\text { accident }\} & =\operatorname{Pr}\{\text { loss }\} . \operatorname{Pr}\{\text { impact } \mid \text { loss }\} \\
& . \operatorname{Pr}\{\text { collision } \mid \text { impact } \cap \text { loss }\} \\
& . \operatorname{Pr}\{\text { damage } \mid \text { collision } \cap \text { impact } \cap \text { loss }\}
\end{aligned}
$$

For the sake of simplicity, the mention to conditionality will be omitted for the rest of the paper by using short notations $\operatorname{Pr}\{$ loss $\}, \operatorname{Pr}\{$ impact $\}, \operatorname{Pr}\{$ collision $\}$ and $\operatorname{Pr}\{$ damage $\}$.

Evaluation of the probability to get an accident for the road network hence requires being able to evaluate each term of expression (1) for all the roads belonging to the network or the region of interest. Models are therefore proposed in the next section.

\section{ROAD NETWORK DATA AND MODELS}

\section{A. Road network data}

Nature and properties of the roads above which the UAV is flying have a strong impact on the risk. Among these properties, the ones of interest are:

- vocation of the road (e.g. motorway, local road, etc.),

- dimensions of the road (number of lanes, width, etc.),

- speed of vehicles on the road (speed average, speed limit, etc.),

- traffic on the road (current speed and number of vehicles)

Although the first three types of properties are time invariant, the last one strongly depends on the day hour.

Getting accurate numerical data for all these properties is not an obvious task, at least for the French road network, because of the dispersion of the sources. The main source used in this paper is the IGN open database [14] which provides information on the location, vocation, number of lanes of all road segments of the French road network. Regarding traffic information, no centralized database exists in France. Some local administrations regularly perform measurements of Annual Average Daily Traffic (AADT) which are provided in open databases. In this paper, some of these databases have been fused to build a representative set of AADT values covering heterogeneous roads in terms of habits and traffic. As each data source does not provide the same level of details, the traffic considered in this paper is assumed to concern only cars. Other vehicles that represent a lower percentage of the traffic (trucks and motorbikes) are not taken into account separately but considered as cars too.

Based on these data sources, traffic and speed limit assumptions have been derived depending on the vocation of the road that is considered (motorway, main liaison, regional liaison and local liaison) and on French regulations. Table 1 presents the values used in this paper. They are representative of the order of magnitude that should be considered, but of course more accurate data should be used according to the geographical scenario of interest because roads with same vocation can have very different traffic habits or speed limits depending on their locations.

The width $l_{r}$ of the considered road segment will be used in the evaluation of $\operatorname{Pr}\{$ impact $\}$ and $\operatorname{Pr}\{$ collision $\}$. It is computed from the number $n_{l r}$ of lanes by assuming a width of 3.5 meters by lane: $l_{r}=3.5^{*} n_{l r}$. 


\begin{tabular}{|ccc|}
\hline Road vocation & $\begin{array}{c}\text { AADT } \\
\text { (veh/day) }\end{array}$ & $\begin{array}{c}\text { Max speed } \\
(\mathbf{k m} / \mathbf{h r})\end{array}$ \\
\hline Motorway & 35000 & 130 \\
\hline Main liaison & 14000 & 90 \\
\hline Regional liaison & 4000 & 70 \\
\hline Local liaison & 2000 & 50 \\
\hline
\end{tabular}

Table 1. Traffic and speed limit depending on road vocation

The speed limit $v_{\max }$ of the vehicles on a road is taken from Table 1 depending on the vocation of the considered road segment. The current speed $v$ and the number $n_{p}$ of vehicles present on the road segment are computed depending the hour $t_{h}$ of the day and AADT value by using the traffic model proposed in Section III-C. They are used in the computation of $\operatorname{Pr}\{$ collision $\}$.

\section{B. Vehicle model}

As previously stated, vehicles considered in this paper are cars. The geometrical vehicle model is exclusively based on an average car represented by a parallelepiped of length $L_{v}=5 \mathrm{~m}$, width $l_{v}=2.5 \mathrm{~m}$ and height $h_{v}=1.7 \mathrm{~m}$. Assuming that vehicles are moving, it is considered that their length is virtually extended by the stopping distance in order to handle stopping dynamics:

$$
L_{m v}(v)=L_{v}+L_{\text {stop }}(v)=L_{v}+L_{p r}(v)+L_{b}(v)
$$

where $L_{\text {stop }}(v)$ is the stopping distance of the vehicle which depends on its speed $v, L_{p r}(v)$ is the distance corresponding to the perception and reaction time of the driver (assumed to be equal to $1 \mathrm{sec})$ and $L_{b}(v)$ is the braking distance both defined in meters by

$$
L_{p r}(v)=\frac{v_{k m / h}}{3.6} \quad L_{b}(v)=\frac{v_{k m / h}{ }^{2}}{254 \cdot f_{r}}
$$

where $f_{r}$ is a friction coefficient taken equal to 0.8 (which corresponds to dry weather conditions) and $v_{k m / h}$ is the speed $v$ of the vehicle expressed in kilometres per hour. Graphical representation of $L_{\text {stop }}(v)$ is presented in Figure 2.

This distance $L_{m v}(v)$ will be used in the traffic model and in the computation of $\operatorname{Pr}\{$ collision $\}$.

\section{Traffic model}

Traffic models can be found in the literature (eg. [11][12]). Most of them may be too complex to be used in a risk analysis method such as the one presented in this paper which requires computations to be run at each time instant of the flight trajectory. Therefore, a simple model is proposed in this paper which aims at being representative enough of traffic tendencies (see for example daily car traffic trends in [13]). This model enables to compute the number $n_{p}$ of vehicles present on a given road segment and their speed $v$, depending on the hour of the day and from available data such as the AADT and the type of road.

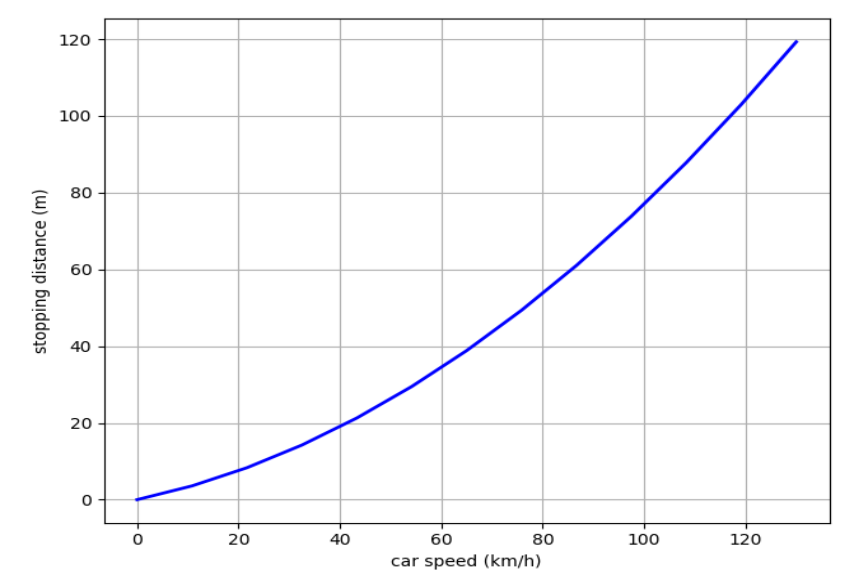

Figure 2. Stopping distance $L_{\text {stop }}$ of a car as a function of its speed

Let $n\left(t_{h}\right)$ denote the instantaneous number of vehicles per time unit present on the road segment $s_{i}$ at time $t_{h}$, defined as a decimal hour $\left(t_{h} \in[0,24]\right)$. It is defined as the sum

$$
n\left(t_{h}\right)=g_{1}^{n}\left(t_{h}\right)+g_{2}^{n}\left(t_{h}\right)
$$

of two Gaussian functions

$$
g_{i}^{n}\left(t_{h}\right)=\lambda \cdot \exp \left(-\frac{1}{2}\left(\frac{t_{h}-\mu_{i}^{t}}{\sigma_{i}^{t}}\right)^{2}\right), \quad i=1,2
$$

where the scaling factor $\lambda$ is computed so as to verify

$$
\int_{t_{h}=0}^{t_{h}=24} n\left(t_{h}\right) d t_{h}=A A D T
$$

and where $\mu_{1}^{t}=8 \mathrm{~h}$ and $\mu_{2}^{t}=18 \mathrm{~h}$ are peak hours for traffic and $\sigma_{1}^{t}=\sigma_{2}^{t}=2 \mathrm{~h}$ standard deviations. An example of graphical representation is given in Figure 3.

The speed $v\left(t_{h}\right)$ of the vehicles is defined in a similar way from a mixture of two Gaussian functions

$$
v\left(t_{h}\right)=g_{1}^{v}\left(t_{h}\right)+g_{2}^{v}\left(t_{h}\right)
$$

with

$$
g_{i}^{v}\left(t_{h}\right)=\left(v_{\max }-v_{\min }\right) \cdot \exp \left(-\frac{1}{2}\left(\frac{t_{h}-\mu_{i}^{t}}{\sigma_{i}^{t}}\right)^{2}\right), \quad i=1,2
$$

where $v_{\max }$ is the maximum speed allowed on the road (hence depending on the type of road as described in Section III-A) and where $v_{\min }$ is an assumption of minimum speed during peak hours and is taken as $v_{\min }=v_{\max } / 3$ in this paper. See Figure 4 for a graphical representation of this speed profile $v\left(t_{h}\right)$.

Note that all these parameters should be adapted to the geographical scenario considered since, for example, traffic in rural or dense urban areas is very different.

The instantaneous number $n\left(t_{h}\right)$ of vehicles per time unit present on the road segment and the speed of vehicles $v\left(t_{h}\right)$ being defined, one can now compute the number $n_{p}\left(t_{h}\right)$ of vehicles being present on the considered road segment at time $t_{h}$. 
This number $n_{p}\left(t_{h}\right)$ of vehicles is simply expressed as

$$
n_{p}\left(t_{h}\right)=n\left(t_{h}\right) \cdot \frac{L_{r}}{v\left(t_{h}\right)}
$$

where $L_{r}$ is the length of the road segment.

Note that $n_{p}\left(t_{h}\right)$ must not be greater than the maximum number $N_{p}{ }^{p}$ ox of vehicles that can be physically present on the road segment

$$
n\left(t_{h}\right) \frac{L_{r}}{v\left(t_{h}\right)}=n_{p}\left(t_{h}\right) \leq N_{p}^{\max }\left(t_{h}\right)=\frac{L_{r}}{L_{m v}}=\frac{L_{r}}{L_{v}+L_{\text {stop }}\left(v\left(t_{h}\right)\right)}
$$

given the length $L_{r}$ of the road segment and the sum of the length of a vehicle and its stopping distance $L_{m v}=L_{v}+L_{\text {stop }}$. The stopping distance is a function of the speed $v$. Since the profile of the speed $v$ has been defined by assigning $v_{\text {min }}$ arbitrarily, it must be verified that this choice will lead to a speed $v\left(t_{h}\right)$ which satisfies equation (10). If not, then $v\left(t_{h}\right)$ is chosen as the solution of equation

$$
n\left(t_{h}\right)\left(L_{v}+L_{\text {stop }}\left(v\left(t_{h}\right)\right)\right)-v\left(t_{h}\right)=0
$$

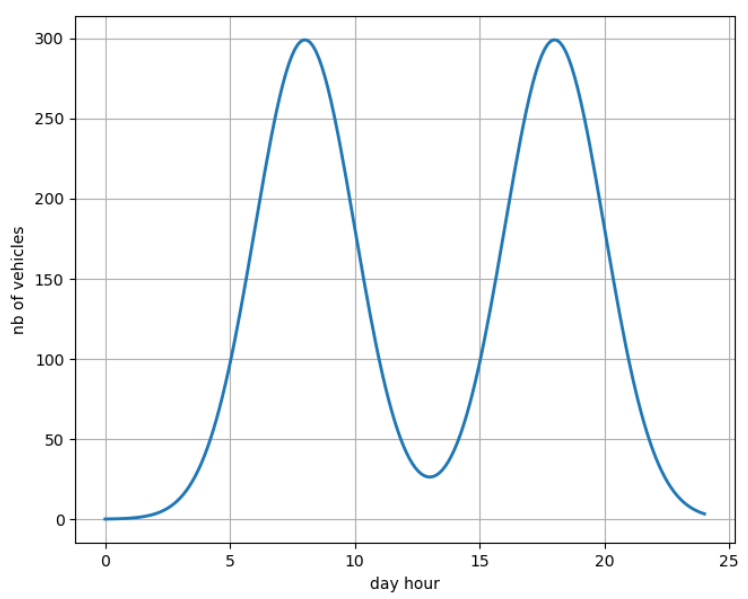

Figure 3. Example of instantaneous number $(n)$ of vehicles per time unit present on the road section as a function of the decimal day hour $\left(t_{h}\right)$ for AADT $=3000$ vehicles per day, $\mu_{l}^{t}=8 \mathrm{~h}, \mu_{2}^{t}=18 \mathrm{~h}$ and $\sigma_{1}^{t}=\sigma_{2}^{t}=2 \mathrm{~h}$

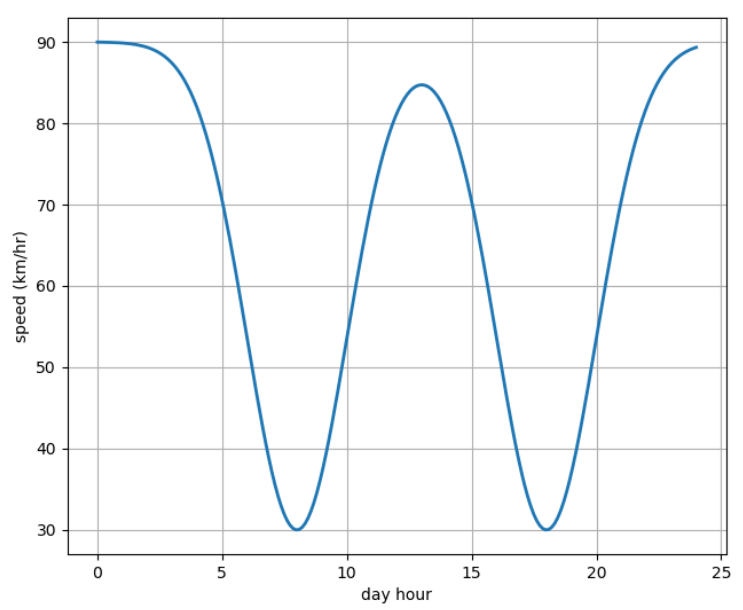

Figure 4. Example of vehicles' speed ( $v)$ as a function of the decimal day hour $\left(t_{h}\right)$ for $v_{\min }=30 \mathrm{~km} / \mathrm{h}, v_{\max }=90 \mathrm{~km} / \mathrm{h}, \mu_{l}^{t}=8 \mathrm{~h}, \mu_{2}^{t}=18 \mathrm{~h}$ and $\sigma_{1}^{t}=\sigma_{2}^{t}=2 \mathrm{~h}$
A simple traffic model has then been defined in this section, making it possible to compute in a tractable way representative values of $v(t)$ and $n_{p}(t)$. These values will be used in the following risk models, e.g. to compute the density of vehicles on the road segment.

\section{RISK MODELS}

In this section, models are proposed to compute each of the terms of expression (1).

\section{A. Loss of control of the UAV}

As in [8] it is assumed that a loss of control of the UAV corresponds to the failure of the main engine, with a probability of occurrence $\operatorname{Pr}\{$ loss $\}=10^{-3}$. This assumption is used only for simplicity reason and corresponds to a first order estimate. Note that a non-controlled descend to the ground can be triggered by different types of critical failures, a collision with another aircraft, a perturbation from the environment, etc. Probabilities of occurrence of these events should hence be taken into account in the computation of $\operatorname{Pr}\{l o s s\}$. Regarding critical failures, a safety analysis of the UAV and of its command and control system should be performed to compute a rate of failure of the whole system to be used in the definition of $\operatorname{Pr}\{$ loss $\}$.

\section{B. Non-controlled ground impact}

Similarly to [8], it is assumed that the maximum range that can be reached by the UAV after loss of control and in a non-controlled descent to the ground corresponds to its glide range (see Figure 5) that is defined by

$$
d_{g}=\frac{-h}{\tan \left(\gamma_{g}\right)}
$$

where $h$ is the ground altitude of the UAV at the time of loss of control. The glide angle $\gamma_{g}$ is computed from the lift-todrag ratio $f$ of the vehicle by

$$
\gamma_{g}=-\tan ^{-1}(f)
$$

The same bivariate normal distribution as in [4][8] is used to model the statistical distribution of the possible impact point, at each possible flight time $t$. This distribution is defined in a reference frame $(G(t), x, y)$ associated to the local ground projection $G$ of the UAV at time $t$ and with main axis $x$ directed along the ground projection of its velocity vector (see Figure 5). In this frame, this distribution, denoted by $f_{\mu, \Sigma}(x, y)$, is defined from its mean vector

$$
\mu=\left[\begin{array}{ll}
0.5 d_{g} & 0
\end{array}\right]^{T}
$$

and covariance matrix

$$
\Sigma=\left[\begin{array}{cc}
\sigma_{x}^{2} & 0 \\
0 & \sigma_{y}^{2}
\end{array}\right] \text { s.t. } \sigma_{x}=\frac{d_{g}}{6} \text { and } \sigma_{y}=\frac{d_{g}}{12}
$$




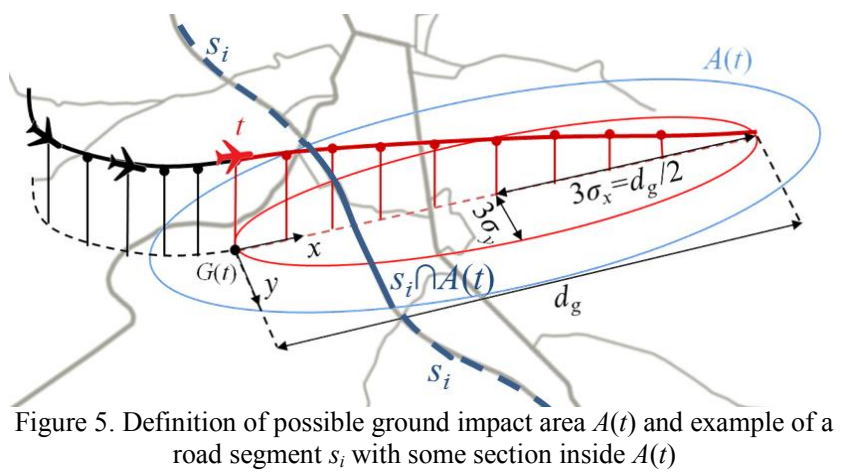

Similarly to [8], the possible ground impact area at time $t$, denoted $A(t)$, is considered to be defined as the "4- $\sigma$ ellipse" associated to this distribution (see Figure 5).

At a given time instant $t$ of the UAV trajectory, and for any road segment $s_{i}$ of the road network, the probability of impact on this road segment is computed as the integral of $f_{\mu, 2}(x, y)$ over the section of $s_{i}$ included in the "4- $\sigma$ ellipse" $A(t)$ :

$$
\operatorname{Pr}\{\text { impact }\}\left(s_{i}, t\right)=\iint_{(x, y) \in s_{i} \cap A(t)} f_{\mu, \Sigma}(x, y) d x d y
$$

\section{Collision with a vehicle}

It is assumed that no dodge is possible to avoid the collision if the UAV falls within the stopping distance of the vehicle.

The probability of collision with a vehicle is computed as the product of the vehicle density $\rho_{i}$ on the considered road segment $s_{i}$ by the surface of collision $S_{c o l}$ between the UAV and a vehicle:

$$
\operatorname{Pr}\{\text { collision }\}\left(s_{i}, t\right)=\rho_{i} * S_{c o l}
$$

The vehicle density $\rho_{i}$ is computed by

$$
\rho_{i}=\frac{n_{p}(t)}{l_{r} \cdot L_{r}}
$$

where $l_{r}$ and $L_{r}$ are respectively the width and length of the road segment and where $n_{p}(t)$ is the number of vehicles present at time $t$ on the road segment, as defined in Section III-C.

The surface of collision depends on the following parameters:

- vehicle dimensions and stopping distance,

- UAV dimensions. Let $r$ denote the radius defined such as $2 * r$ corresponds to the maximum dimension of the UAV.

- gliding angle $\gamma_{g}$ of the UAV,

- collision angle $\alpha_{c o l}$ defined as the relative azimuth between the vehicle flow direction and the ground projection of the velocity vector of the UAV.

Note that the collision angle should take into account the flow direction of the vehicles on the road and therefore should be defined on $[0,2 \pi]$. In this paper, a conservative

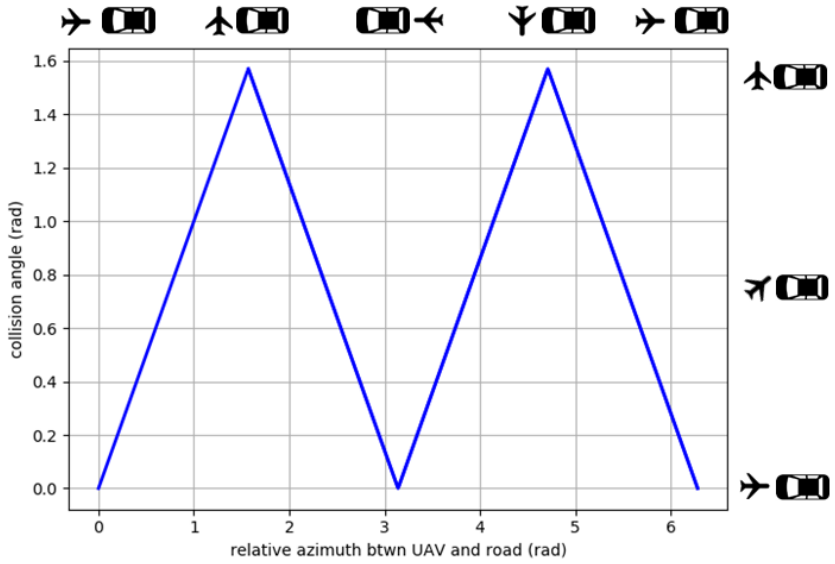

Figure 6. Definition of worst case collision angle $\alpha_{c o l}$ as a function of the relative azimuth angle between the UAV and the vehicle flow

approach is taken by defining $\alpha_{c o l}$ on $[0, \pi / 2]$ which corresponds to a worst case of a collision configuration close to opposite directions (see Figure 6). This choice could have an impact on the relative kinetic energy and then on $\operatorname{Pr}\{$ damage $\}$ since it clearly depends on the value of this collision angle (see next section).

To evaluate the collision surface $S_{c o l}$, let us first introduce the surface $S_{c o l}^{l}$ defined as

$$
S_{c o l}^{1}=S_{c o l}^{e}+S_{c o l}^{r}+2 \cdot S_{c o l}^{p}
$$

where the three surfaces $S_{c o l}^{e}, S_{c o l}^{r}$ and $S_{c o l}^{p}$ are defined in

Figure 7 and computed as follows

$$
\begin{gathered}
S_{c o l}^{e}=l_{v} \cdot L_{m v}+2 r \cdot l_{v}+2 r \cdot L_{m v}+\pi r^{2} \\
S_{c o l}^{r}=2 r \cdot d \\
S_{c o l}^{p}=d \cdot\left(\frac{L_{m v}}{2} \sin \alpha_{c o l}+\frac{l_{v}}{2} \cos \alpha_{c o l}\right)
\end{gathered}
$$

with

$$
d=\frac{-h_{v}}{\tan \left(\gamma_{g}\right)}
$$

Contrary to what is done in the case of risk evaluation for people at ground, where the population density can be assumed, for the computation of $\operatorname{Pr}\{$ collision $\}$, to be locally homogenous over all the collision surface between the UAV and someone, this assumption cannot hold here. Indeed, as vehicles cannot be located outside the road, assigning $S_{c o l}=S_{c o l}^{l}$ would lead to an overestimation of $\operatorname{Pr}\{$ collision $\}$ for cases where the surface of collision is greater than the road section located inside it. Let us denote $S_{r}$ the intersection of the road and the surface $S_{c o l}^{l}$. This surface is computed as:

$$
\begin{aligned}
& - \text { if } \alpha_{c o l} \neq 0 \text { : } \\
& \quad S_{r}=\left(2\left(\frac{L_{m v}}{2} \sin \alpha_{c o l}+\frac{l_{v}}{2} \cos \alpha_{c o l}\right)+2 r\right) \cdot \frac{l_{r}}{\cos \left(\frac{\pi}{2}-\alpha_{c o l}\right)}
\end{aligned}
$$



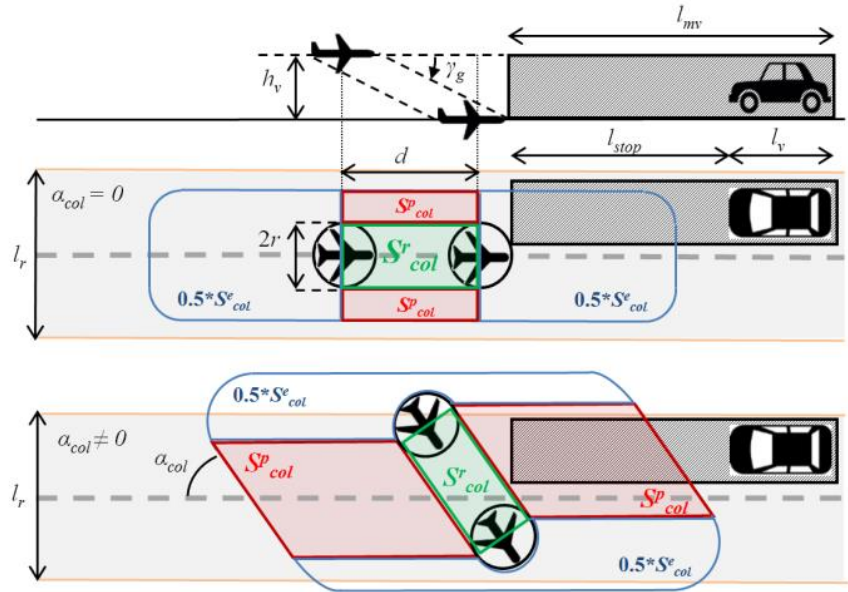

Figure 7. Collision area definition

- if $\alpha_{c o l}=0$ :

$$
S_{r}=l_{r} \cdot\left(d+2 r+L_{m v}\right)
$$

Finally, the surface of collision used in the evaluation of $\operatorname{Pr}\{$ collision $\}$ is computed as

$$
S_{c o l}=\min \left(S_{c o l}^{1}, S_{r}\right)
$$

Figure 8 and Figure 9 illustrate the effect of the proposed saturation approach on the computation of the surface of collision. In the case with no saturation $\left(S_{c o l}=S_{c o l}^{l}\right)$ it can be observed on Figure 8 that $S_{c o l}$ first increases with the collision angle (for $0^{\circ} \leq \alpha_{c o l}<70^{\circ}$ ) and then decreases with the collision angle (for $\alpha_{c o l} \geq 70^{\circ}$ ). Since no saturation is applied, it can be seen that the road width has no influence. In the case with saturation, as defined by equation (26), the same behaviour holds for values of road width and collision angle below some "saturation level" represented in white on Figure 9. For a given value of the road width, when the collision angle becomes greater than this saturation level, the surface of collision $S_{c o l}$ also starts to decrease.

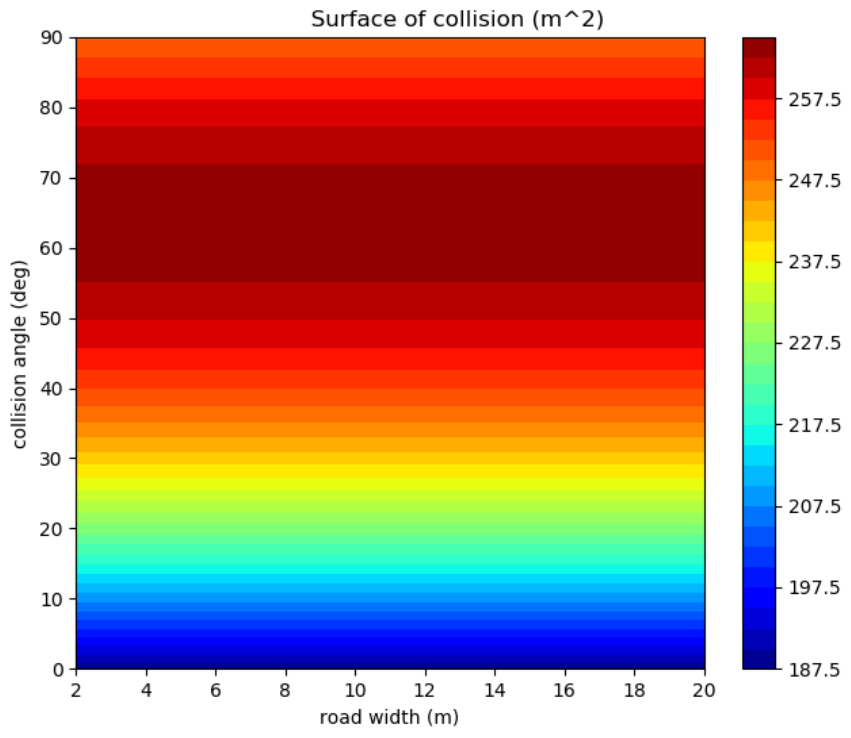

Figure 8. Surface of collision with respect to collision angle and road width without the saturation approach $\left(S_{c o l}=S_{c o l}^{l}\right)$

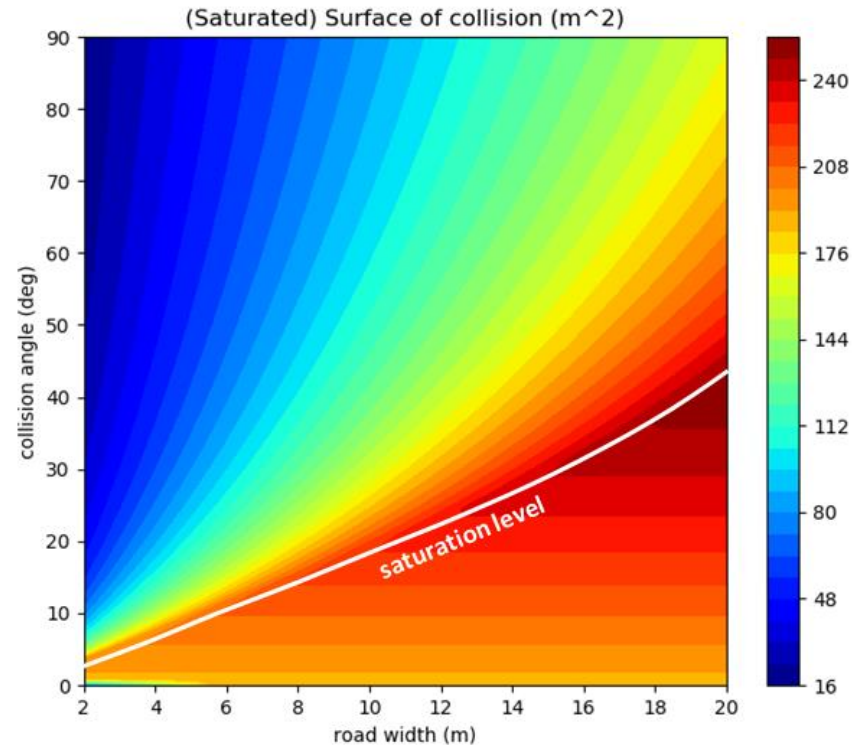

Figure 9. Surface of collision with respect to collision angle and road width with the saturation approach $\left(S_{c o l}=\min \left(S_{c o l}^{l}, S_{r}\right)\right)$

\section{Damage}

A conservative approach considering $\operatorname{Pr}\{$ damage $\}=1$ is chosen in this paper. If one would like to investigate the influence of some risk mitigation procedure (e.g. flight termination systems such as emergency parachute) it is important to dispose of a model enabling to evaluate $\operatorname{Pr}\{$ damage $\}$. The derivation of such a model is beyond the scope of this paper and left for future work. Nevertheless, it is worth noticing that damage to the vehicle and/or to its passengers would mainly depend on the kinetic energy of impact and on the impact angle between the UAV and the vehicle. Some assumption on the evolution of $\operatorname{Pr}\{$ damage $\}$ with respect to the kinetic energy of impact could be done, in the case of damage to vehicle or to passengers, e.g. based on sigmoid functions as reviewed in [10].

\section{EVALUATION EXAMPLE}

In this section, a simple example is introduced to illustrate the use of the proposed risk models in the evaluation of the risk of getting an accident for a user of a road network due to a fixed-wing UAV in operation.

The road network illustrated in Figure 10 is composed of 5 motorway segments, 21 road segments with "main liaison" vocation and 27 road segments with "local liaison" vocation. The width of the plotted lines on the figure is representative of the width of the road. The trajectory of the UAV is represented in blue on the same figure, the initial position of the UAV being represented by a blue dot. The flight parameters and characteristics of the UAV are given in Table 2. They are representative of a typical fixed-wing UAV that could be used for Long Range Operations such as railways or power lines inspection. The trajectory along which the risk is evaluated in this section is a small portion of a longer trajectory that would also be representative of such monitoring missions. 


\begin{tabular}{|ccc|}
\hline UA V characteristics & Value & Unit \\
\hline max radius $(r)$ & 3.3 & $\mathrm{~m}$ \\
\hline ground altitude $(h)$ & 130 & $\mathrm{~m}$ \\
\hline lift over drag ratio $(f)$ & 12 & $(-)$ \\
\hline speed & 14 & $\mathrm{~m} . \mathrm{s}^{-1}$ \\
\hline
\end{tabular}

Table 2. UAV and flight main characteristics

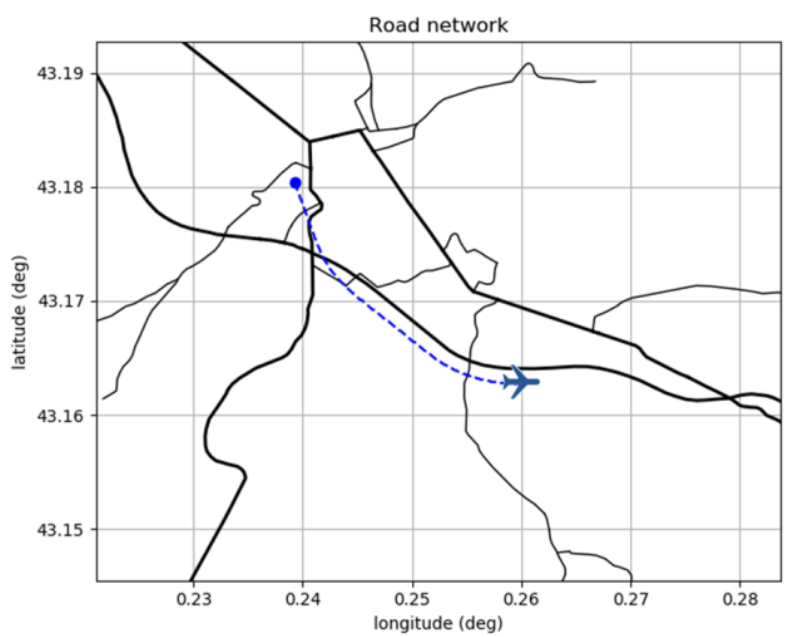

Figure 10. Roadnetwork considered for risk analysis (black) and UAV trajectory (blue dot is the initial position)

\section{RISK ASSESSMENT}

\section{A. Evaluation and metrics}

Evaluation of the risk is achieved by computing all the terms of equation (1) at each flight instant $t$, for all the road segments that belong to the possible ground impact area $A(t)$. To be able to perform some risk analysis, several metrics are considered, based on adaptations of the ones introduced in [8]. They are presented below using the generic notation $\operatorname{Pr}\{$.$\} to refer to any of the terms of the risk evaluation$ expression (1):

- $\quad$ risk statistics: the statistics (min, max, med, mean and std dev values) of each $\operatorname{Pr}\{$.$\} is computed over$ all road segments included in any possible impact area $A(t)$ for all time instants $t$ of the trajectory.

- $\quad$ instantaneous maximum probability $\left(\mathrm{Pr}_{\text {inst }}^{\max }\right)$ : at each time instant $t$ of the flight trajectory, the maximum of $\operatorname{Pr}\{$.$\} is computed over the corresponding$ possible impact area $A(t)$ :

$\forall t, \operatorname{Pr}_{\text {inst }}^{\max }\{\}.(t)=\operatorname{Max}\left\{\operatorname{Pr}\{\cdot\}\left(s_{i}, t\right) \mid i=1, . ., N(t)\right\}$

where $N(t)$ is the number of road segments $s_{i}$ at time $t$ which are included in the possible impact area $A(t)$.

- spatial maximum probability $\left(\mathrm{Pr}_{s p a}^{\max }\right)$ : for each possible road segment $s_{i}$ the maximum of $\operatorname{Pr}\{$.$\} is$ computed over the whole trajectory duration:

$$
\forall s_{i} \in A^{*}, \operatorname{Pr}_{s p a}^{\max }\{.\}\left(s_{i}\right)=\operatorname{Max}\left\{\operatorname{Pr}\{.\}\left(s_{i}, t\right) \mid t\right\}
$$

where $A^{*}$ stands for the set of all the $A(t)$ over the flight trajectory.

- risk exposition time $\left(T_{\text {exp }}\right)$ : for each possible road segment $s_{i}$ included in $A^{*}$, the time duration it belongs to any potential impact area $A(t)$, for all $t$ over the flight trajectory, is computed.

Based on the risk exposition time $T_{\exp }$ and on $\underset{\text { spa }}{\max }\{$ accident $\}$, a categorization into several classes can be performed as it can be assumed that road segments with small probability of accident but high risk exposition time could be considered as risky as road segments with high probability of accident but small risk exposition time. Such a classification has been proposed in [8] and can of course be used in this paper. Another approach is chosen here for the sake of simplicity, which consists in computing the following risk index $I_{r}$ for each road segment

$$
\forall s_{i} \in A^{*}, I_{r}\left(s_{i}\right)=T_{\text {exp }}\left(s_{i}\right) \cdot \operatorname{Pr}_{s p a}^{\max }\{\cdot\}\left(s_{i}\right)
$$

It has no physical meaning but it would enable to quickly distinguish road segments that should be considered as very risky (i.e. high risk exposition time and high probability of accident leading to a high value for $I_{r}$ ), from low risky (small value for $I_{r}$ ) and medium risky.

An example of risk evaluation and analysis based on these metrics is presented in the next subsection.

\section{B. Example of evaluation}

Evaluation has been performed for the scenario introduced in Section V, at time $t_{h}=8 \mathrm{~h}$ corresponding to a traffic peak hour.

As can be seen on Figure 11, the probability $\underset{\operatorname{Pr}_{\text {spa }}}{\max }\{$ impact $\}$ is greater over road segments for which there exist time instants at which they are located close to the center of the impact

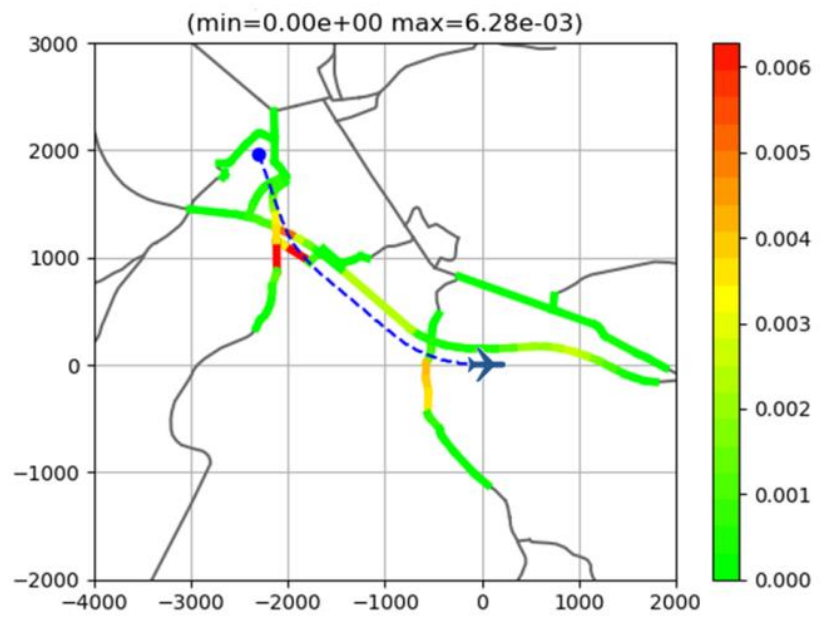

Figure 11. Geographical representation of $\underset{\operatorname{Pr}}{\max }$ mampact $\}$ along the flight trajectory (local metric coordinates) 


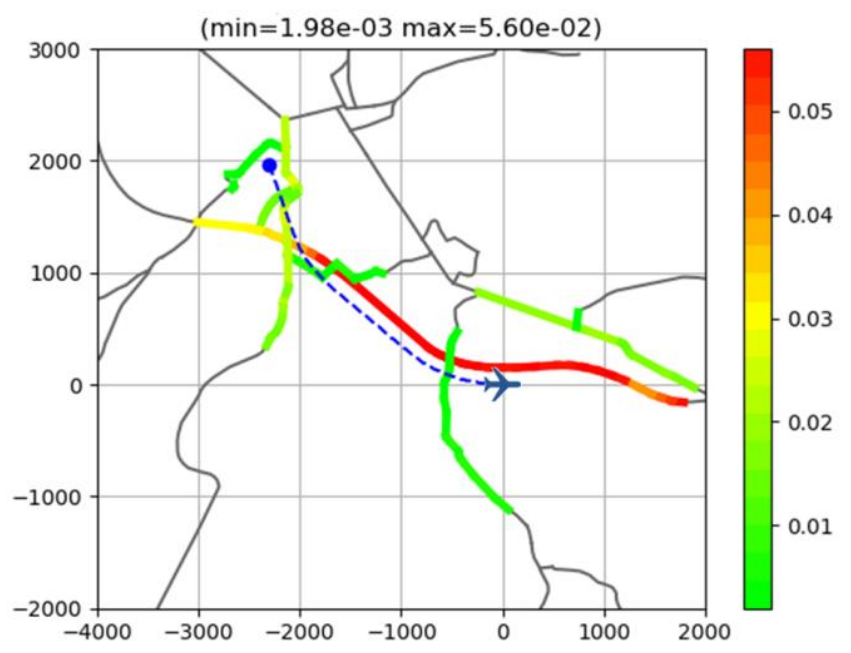

Figure 12. Geographical representation of $\underset{\operatorname{Pr}}{\max }\{$ collision $\}$ along the flight trajectory (local metric coordinates)

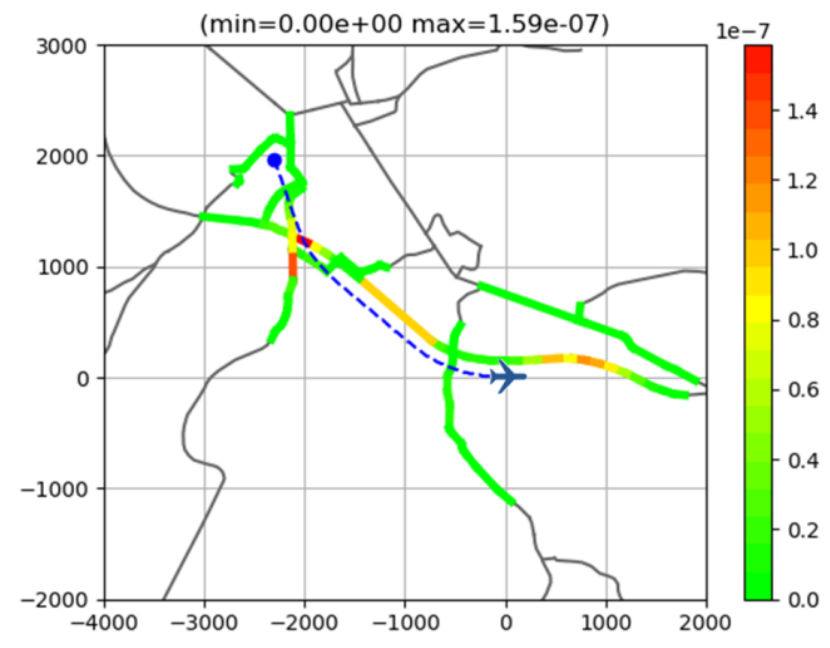

Figure 13. Geographical representation of $\underset{\operatorname{Pr}}{\max a}$ \{accident $\}$ along the flight trajectory (local metric coordinates)

areas (see Section IV-B). For $\underset{\operatorname{Pr}}{\max }$ max $\{$ collision $\}$, greater values correspond to road segments with important traffic and with respect to which the collision angle is small (i.e. flight trajectory parallel to the road, see Figure 12).

The evaluation of expression (1) enables to compute $\operatorname{Pr}_{\text {spa }}^{\max }\{$ accident $\}$ which is plotted on Figure 13. Road segments with high risk are the ones for which the impact and collision terms are also simultaneously high.

Risk exposition time metric has also been computed for this illustration scenario. It is plotted on Figure 14. Road segments with high values of $T_{\exp }$ are the ones which correspond to straight sections of the flight trajectory. Road segments located close to the beginning or to the end of the considered flight trajectory exhibit smaller values for $T_{\text {exp }}$ since they belong to a potential crash area during a more limited duration.

The values of the risk index $I_{r}$ are plotted on Figure 15. In the considered illustration scenario, one road segment (in red in Figure 15) should therefore be considered as highly risky and two smaller road segments (in orange in Figure 15) should be assigned to a medium risk class. This information can be easily exploited and helpful for mission preparation to quickly identify risky road segments and modify the flight plan accordingly, or for flight authorizations to verify the compliance of a proposed flight plan to a risk level requirement.

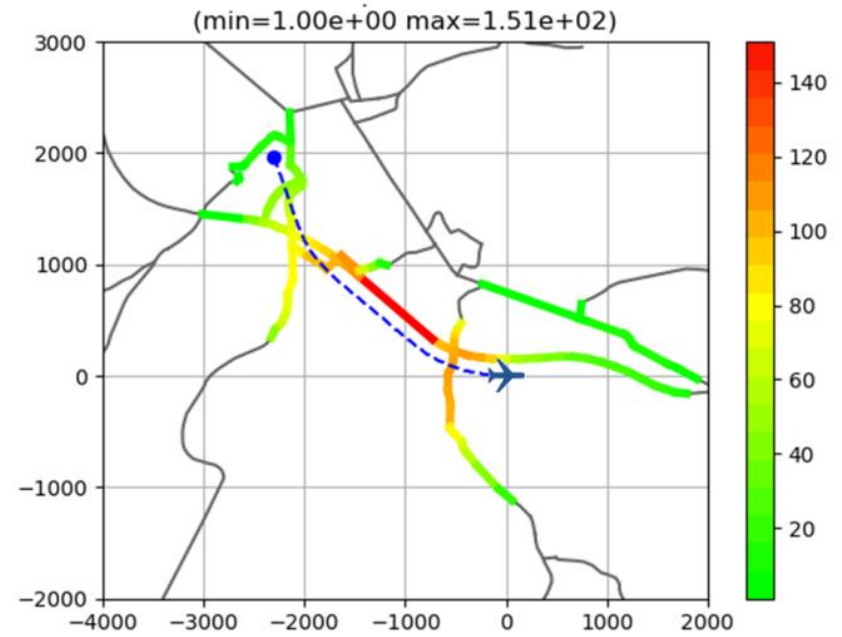

Figure 14. Geographical representation of the risk exposition time $T_{\text {exp }}$ (in seconds) along the flight trajectory (local metric coordinates)

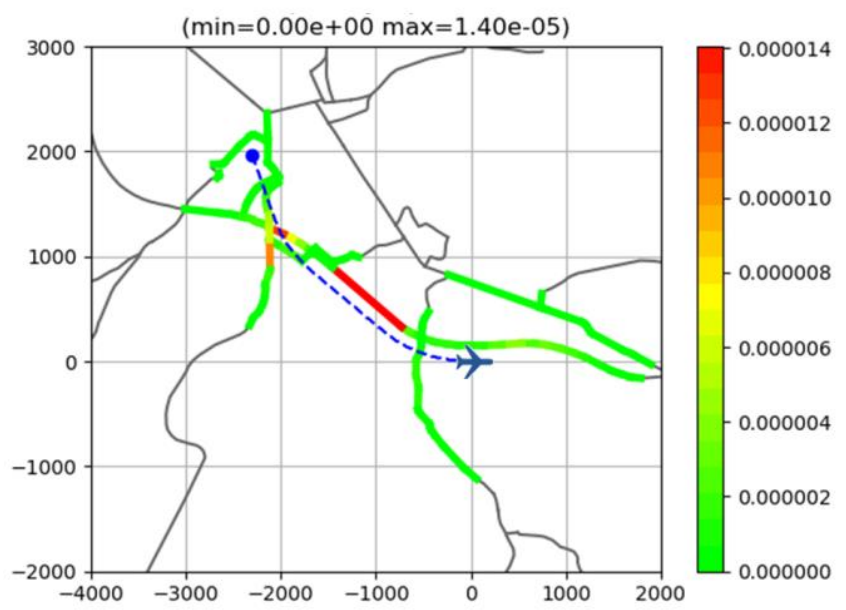

Figure 15. Geographical representation of the risk index $I_{r}$ along the flight trajectory (local metric coordinates)

\section{Sensitivity to day hour $t_{h}$}

To illustrate the influence of the day hour $t_{h}$ on the results, several risk evaluations have been run for the same scenario but considering different values of $t_{h}$ in $[0,24]$. The value of $\mathrm{Pr}_{\text {inst }}^{\max }\{$ accident $\}$ is plotted with respect to $t_{h}$ in Figure 16. As expected, the influence of the traffic can be easily observed. 


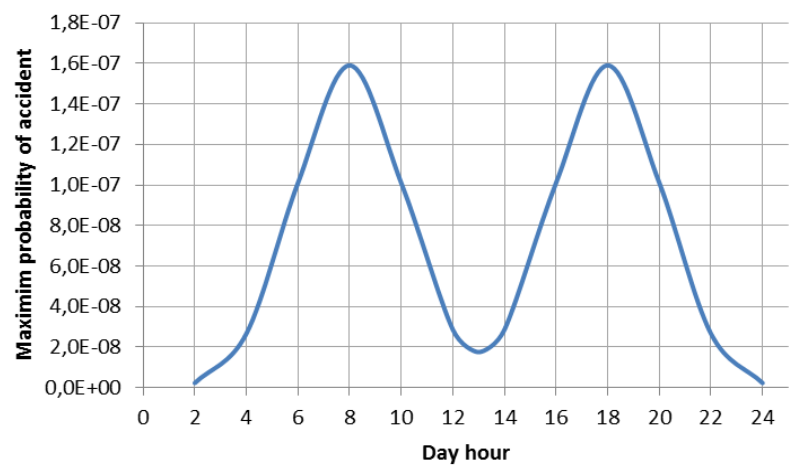

Figure 16. Influence of day hour $t_{h}$ on $\operatorname{Pr}_{\text {inst }}^{\max }\{$ accident $\}$

A difference of one order of magnitude is obtained for this maximum risk value between peak hours $\left(\sim 1.6^{*} 10^{-7}\right)$ and hours with low traffic $\left(\sim 2 * 10^{-8}\right)$.

Therefore it is of a huge importance to be able to validate or adapt the parameters of the traffic model to precise traffic data representative of the local geographical scenario, when available, if absolute risk levels have to be computed. If not, this proposed approach should rather be used in a comparative way, to decrease the sensitivity to traffic data, to identify for example the influence of the UAV trajectory or of mitigation risk procedures if their effects can be included in the models.

\section{CONCLUSION}

In this paper, models have been proposed for risk assessment of UAV operations over road networks. They enable to compute the probability to get an accident for a user of the road network due to the crash of a fixed-wing UAV on a road. A simple traffic model has been proposed to be able to introduce sensitivity of the proposed models to the type of road and time of the day. An example of risk assessment making use of these models has been proposed by considering a simulation example on a road network of several kilometres. The computation of all the probabilities involved in the risk evaluation have been illustrated and a simple risk index has been introduced to help identifying main risky areas, based on probability of accident and risk exposition time.

Future work will address the improvement of the models, including more accurate prediction of ground impact area, and the use of these models in combination with risk assessment for people of inhabited areas. Being able to perform such combined analyses may be helpful in the regulation and flight authorization process and can be considered as a prerequisite to a wide and operational use of UAVs for long range and BVLOS (Beyond Visual Line of Sight) operations. Future work will also consider model uncertainties and the study of their impact on the evaluation precision.

\section{REFERENCES}

[1] H. Blom, B. Bakker, M. Everdij and M. Van der Park, "Collision Risk Modelling of Air Traffic", European Control Conference, Cambridge, UK, 2003.

[2] R. E. Weibel and R. J. Hansman, "Safety Consideration for Operation of Different Classes of UAVs in the NAS", AIAA's 3rd Unmanned Unlimited Technical Conference, Workshop and Exhibit, 2004.

[3] D. W. King, A. Bertapelle and C. Mose, "UAV Failure Rate Criteria for Equivalent Level of Safety", International Helicopter Safety Symposium, 2005.

[4] R. Clothier, R. Walker, N. Fulton and D. Campbell, "A Casualty Risk Analysis for Unmanned Aerial System (UAS) Operations over Inhabited Areas," 12th Australian International Aerospace Congress, 2007.

[5] K. Dalamagkidis, K. P. Valavanis and L. A. Piegl, "Evaluating the Risk of Unmanned Aircraft Ground Impacts", 16th Mediterranean Conference on Control and Automation, 2008.

[6] R. Melnyk, D. Schrage, V. Volovoi and H. Jimenez, "A Third-Party Casualty Risk Model for Unmanned Aircraft System Operations", Reliability Engineering and System Safety, vol. 124, pp. 105-116, 2014.

[7] A. la Cour-Harbo, "Quantifying Risk of Ground Impact Fatalities of Power Line Inspection BVLOS Flight with Small Unmanned Aircraft", International Conference on Unmanned Aircraft Systems, Miami, USA, 2017.

[8] S. Bertrand, N. Raballand, F. Viguier and F. Muller, "Ground Risk Assessment for Long-Range Inspection Missions of Railways by UAVs", International Conference on Unmanned Aircraft Systems, Miami, USA, 2017.

[9] K. Bhalla, M. Ezzati, A. Mahal, J. Salomon and M. Reich, "A RiskBased Method for Modelling Traffic Fatalities", in Risk Analysis, vol. 27, no. 1, pp. 125-135, 2007.

[10] C. Jurewicz, A. Sobhani, J. Woolley, J. Dutschke and B. Corben, "Exploration of Vehicle Impact Speed - Injury Severity Relationships for Application in Safer Road Design", in Transportation Research Procedia, vol. 14, pp. 4247-4256, 2016.

[11] E. Thonhofer, M. Fuhrmann and S. Jakubek, "Parameter Identification and Model Validation of a Macroscopic Traffic Model", in IFAC Papers Online, vol. 50, no.1, pp. 12835-12840, 2017.

[12] F. Morbidi, L. L. Ojeda, C. Canudas de Wit and I. Bellicot, "A New Robust Approach for Highway Traffic Density Estimation”, European Control Conference, Strasbourg, France, 2014.

[13] A Heyworth and R. German, "Road Traffic Estimates in Great Britain: 2016 Report", Statistical Release of Annual Traffic Estimates, UK Department for Transport, 2017.

[14] "Route 500" database, IGN (Institut National de l'Information Géographique et Forestière), http://professionnels.ign.fr/route500 